We have presented the Graphical Abstract text and image for your article below. This brief summary of your work will appear in the contents pages of the issue in which your article appears.

1

\section{Biodegradable zwitterionic nanoparticles with tunable UCST-type phase separation under physiological conditions}

Mattia Sponchioni, Paola Rodrigues Bassam, Davide Moscatelli, Paolo Arosio and Umberto Capasso Palmiero*

We report tunable biodegradable zwitterionic nanoparticles with UCST behavior under physiological conditions that can be used in controlled delivery strategies.

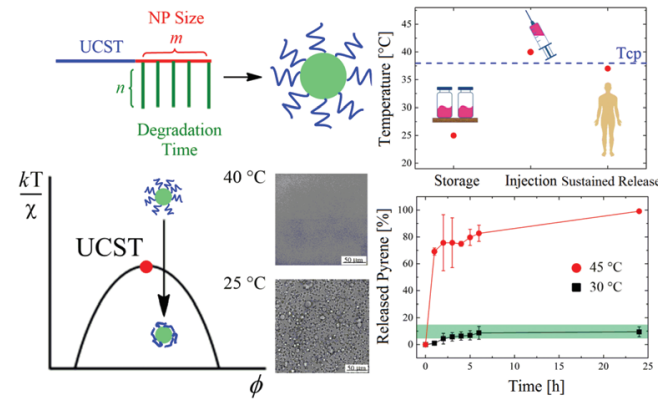

Please check this proof carefully. Our staff will not read it in detail after you have returned it.

Please send your corrections either as a copy of the proof PDF with electronic notes attached or as a list of corrections. Do not edit the text within the PDF or send a revised manuscript as we will not be able to apply your corrections. Corrections at this stage should be minor and not involve extensive changes.

Proof corrections must be returned as a single set of corrections, approved by all co-authors. No further corrections can be made after you have submitted your proof corrections as we will publish your article online as soon as possible after they are received.

Please ensure that:

- The spelling and format of all author names and affiliations are checked carefully. You can check how we have identified the authors' first and last names in the researcher information table on the next page. Names will be indexed and cited as shown on the proof, so these must be correct.

- Any funding bodies have been acknowledged appropriately and included both in the paper and in the funder information table on the next page.

- All of the editor's queries are answered.

- Any necessary attachments, such as updated images or ESI files, are provided.

Translation errors can occur during conversion to typesetting systems so you need to read the whole proof. In particular please check tables, equations, numerical data, figures and graphics, and references carefully.

Please return your final corrections, where possible within $\mathbf{4 8}$ hours of receipt, by e-mail to: nanoscalearsc.org. If you require more time, please notify us by email. 


\section{Funding information}

Providing accurate funding information will enable us to help you comply with your funders' reporting mandates. Clear acknowledgement of funder support is an important consideration in funding evaluation and can increase your chances of securing funding in the future.

We work closely with Crossref to make your research discoverable through the Funding Data search tool (http://search. crossref.org/funding). Funding Data provides a reliable way to track the impact of the work that funders support. Accurate funder information will also help us (i) identify articles that are mandated to be deposited in PubMed Central (PMC) and deposit these on your behalf, and (ii) identify articles funded as part of the CHORUS initiative and display the Accepted Manuscript on our web site after an embargo period of 12 months.

Further information can be found on our webpage (http://rsc.li/funding-info).

\section{What we do with funding information}

We have combined the information you gave us on submission with the information in your acknowledgements. This will help ensure the funding information is as complete as possible and matches funders listed in the Crossref Funder Registry.

If a funding organisation you included in your acknowledgements or on submission of your article is not currently listed in the registry it will not appear in the table on this page. We can only deposit data if funders are already listed in the Crossref Funder Registry, but we will pass all funding information on to Crossref so that additional funders can be included in future.

\section{Please check your funding information}

The table below contains the information we will share with Crossref so that your article can be found via the Funding Data search tool. Please check that the funder names and grant numbers in the table are correct and indicate if any changes are necessary to the Acknowledgements text.

\begin{tabular}{|l|l|l|l|}
\hline Funder name & $\begin{array}{l}\text { Funder's main country } \\
\text { of origin }\end{array}$ & $\begin{array}{l}\text { Funder ID } \\
\text { (for RSC use only) }\end{array}$ & Award/grant number \\
\hline $\begin{array}{l}\text { Eidgenössische Technische } \\
\text { Hochschule Zürich }\end{array}$ & Switzerland & 501100003006 & Unassigned \\
\hline
\end{tabular}

\section{Researcher information}

Please check that the researcher information in the table below is correct, including the spelling and formatting of all author names, and that the authors' first, middle and last names have been correctly identified. Names will be indexed and cited as shown on the proof, so these must be correct.

If any authors have ORCID or ResearcherID details that are not listed below, please provide these with your proof corrections. Please ensure that the ORCID and ResearcherID details listed below have been assigned to the correct author. Authors should have their own unique ORCID iD and should not use another researcher's, as errors will delay publication.

Please also update your account on our online manuscript submission system to add your ORCID details, which will then be automatically included in all future submissions. See here for step-by-step instructions and more information on author identifiers.

\begin{tabular}{|l|l|l|l|}
\hline First (given) and middle name(s) & Last (family) name(s) & ResearcherID & ORCID iD \\
\hline Mattia & Sponchioni & & $0000-0002-8130-6495$ \\
\hline Paola & Rodrigues Bassam & & \\
\hline Davide & Moscatelli & G-8795-2013 & $0000-0003-2759-9781$ \\
\hline Paolo & Arosio & & $0000-0002-2740-1205$ \\
\hline Umberto & Capasso Palmiero & & $0000-0002-9683-491 X$ \\
\hline
\end{tabular}




\section{Queries for the attention of the authors}

\section{Journal: Nanoscale Paper: c9nr04311j}

Title: Biodegradable zwitterionic nanoparticles with tunable UCST-type phase separation under physiological conditions

For your information: You can cite this article before you receive notification of the page numbers by using the following format: (authors), Nanoscale, (year), DOI: 10.1039/c9nr04311j.

Editor's queries are marked like this Q1, Q2, and for your convenience line numbers are indicated like this $5,10,15, \ldots$

Please ensure that all queries are answered when returning your proof corrections so that publication of your article is not delayed.

\begin{tabular}{|c|c|c|}
\hline $\begin{array}{l}\text { Query } \\
\text { Reference }\end{array}$ & Query & Remarks \\
\hline Q1 & $\begin{array}{l}\text { Funder details have been incorporated in the funder table using } \\
\text { information provided in the article text. Please check that the } \\
\text { funder information in the table is correct. }\end{array}$ & \\
\hline Q2 & $\begin{array}{l}\text { Please confirm that the spelling and format of all author names } \\
\text { is correct. Names will be indexed and cited as shown on the } \\
\text { proof, so these must be correct. No late corrections can be } \\
\text { made. }\end{array}$ & \\
\hline Q3 & $\begin{array}{l}\text { Ref. } 17 \text { and 29: Can these references be updated? If so, please } \\
\text { provide the relevant information such as year, volume and page } \\
\text { or article numbers as appropriate. }\end{array}$ & \\
\hline Q4 & Ref. 32: Please provide the journal title. & \\
\hline Q5 & $\begin{array}{l}\text { Ref. 42, } 44 \text { and } 48 \text { : Please check that the name "U. Capasso } \\
\text { Palmiero" author is displayed correctly. }\end{array}$ & \\
\hline
\end{tabular}




\title{
Biodegradable zwitterionic nanoparticles with tunable UCST-type phase separation under physiological conditions $\uparrow$
}

\author{
Mattia Sponchioni, (D) ${ }^{\text {a,b }}$ Paola Rodrigues Bassam, ${ }^{\mathrm{b}}$ Davide Moscatelli, (D) ${ }^{\mathrm{b}}$ \\ Paolo Arosio (D) a and Umberto Capasso Palmiero (D) *a
}

Thermo-responsive polymeric nanoparticles (NPs) are emerging as a powerful tool in nanomedicine for the fabrication of advanced drug delivery systems. In addition to their size and biodegradation rate, phase separation of NPs upon applying a thermal stimulus provides an additional switch to control the rate of release of active components. Among the materials currently developed for biomedical applications, NPs stabilized by zwitterionic polymers are gaining increasing interest due to their high stability and ability to escape the body immune response. Yet, biodegradable zwitterionic NPs with temperature response under physiological conditions are currently not available. Here, we develop a new class of biodegradable zwitterionic NPs that exhibit UCST phase transition in the biological temperature range $\left(T=30-45^{\circ} \mathrm{C}\right)$ and in physiological solution (i.e. $0.9 \% \mathrm{w} / \mathrm{w} \mathrm{NaCl}$ ). We design a strategy that relies on the self-assembly of block copolymers produced via reversible addition-fragmentation chain transfer (RAFT) emulsion polymerization. These copolymers comprise a zwitterionic portion exhibiting an upper critical solution temperature (UCST) and a biodegradable hydrophobic block consisting of oligoesters functionalized with a vinyl group. This modular macromolecular architecture allows us to independently control a variety of NP properties by modifying the individual components of the copolymer. In particular, the zwitterionic block of the copolymers controls the UCST-type phase separation behavior, while the number of the oli-

Received 20th May 2019, Accepted 13th August 2019

DOI: $10.1039 /$ c9nr04311j

rsc.li/nanoscale goester repeating units governs the size of the NPs and the length of the oligoester dictates the degradation rate. After demonstrating the synthesis of highly controlled degradable NPs, we show the potential of this new class of materials in the context of drug delivery by controlling the release of a drug-mimic molecule upon temperature variations in a broad time range from few minutes to 20 hours.

\section{Introduction}

${ }^{a}$ Department of Chemistry and Applied Biosciences, Institute for Chemical and Bioengineering, ETH Zurich, Vladimir-Prelog-Weg 1, 8093 Zurich, Switzerland. E-mail: umberto.capasso@chem.ethz.ch

${ }^{b}$ Department of Chemistry, Materials and Chemical Engineering "Giulio Natta", Politecnico di Milano, Via Mancinelli 7, 20131 Milano, Italy

$\dagger$ Electronic supplementary information (ESI) available. See DOI: 10.1039/ c9nr04311j responsive materials are generally less affected by the presence of biological macromolecules and electrolytes.

Thermo-responsive polymers are commonly divided with respect to their solubility above or below the threshold temperature. Polymers with a lower critical solution temperature (LCST) are miscible with the solvent in the region of the phase diagram (i.e. temperature vs. polymer volume fraction) below the binodal curve, the LCST being the minimum of this curve. In contrast, polymers with an upper critical solution temperature (UCST) are miscible with the solvent above their binodal curve, the UCST being the maximum of this curve. ${ }^{9-11}$ In particular, temperature-responsive polymer-water mixtures exhibiting an UCST exist as a solution above the transition temperature and phase separate into a polymer rich phase below it. ${ }^{11}$ This critical temperature is often referred to as the cloud point $\left(T_{\mathrm{cp}}\right)$, since the formation of a polymer-rich globular phase leads to a variation in the refractive index and, hence, to the cloudiness of the system.

LCST polymers typically rely on water entropy variations that are poorly affected by the physiochemical characteristics 
of synthetic polymers. In contrast, UCST polymers need to exhibit strong intermolecular enthalpic interactions, either via hydrogen bonding or via electrostatic interactions. ${ }^{12-15}$ These forces are more challenging to encode in synthetic polymers and are more sensitive to environmental factors. As a consequence, UCST polymers are less common compared to the LCST counterparts. ${ }^{16}$ Yet, their development is urgently needed in many biomedical applications where the phase separation induced upon heating is a detrimental process that may lead to premature degradation of encapsulated, conjugated or adsorbed biotherapeutics such as thermolabile small molecules, proteins, exosomes, or cells. ${ }^{11,17-19}$ Promising UCST polymers currently developed include poly(acrylamideco-acrylonitrile)-based copolymers, ${ }^{20-23}$ poly( $N$-acryloyl glycinamide) (PNAGA) ${ }^{24,25}$ ureido derivatized polymers, ${ }^{26-29}$ and zwitterionic polymers.

For the latter ones, the occurrence of the UCST is driven by the intra-chain and/or inter-chain pairing of the zwitterionic groups (e.g. ammonium cations and sulfo-anions in the case of polysulfobetaine). ${ }^{30,31}$ Among the betaine-based polymers, one of the most studied examples is poly([2-(methacryloyloxy) ethyl]dimethyl-(3-sulfopropyl)ammonium hydroxide) (pSB) mainly because of its biocompatibility and thermoresponsiveness. $^{32,33}$ The transition temperature of this polymer in aqueous solution is strongly dependent on the concentration, molecular weight, ${ }^{34,35}$ branching density, ${ }^{35}$ presence of salts, and spacing between ionic units. ${ }^{36}$ In particular, the addition of low molar mass electrolytes has been observed to significantly decrease the zwitterionic polymer UCST to values lower than the physiological range $\left(30-45^{\circ} \mathrm{C}\right)$, in some cases even lower than the water freezing point, according to the so called "antipolyelectrolyte effect". ${ }^{37}$ This feature unfortunately prevents the application of these polymers in biological systems.

There is therefore a severe lack of zwitterionic polymers suitable for the production of NPs aimed at biomedical applications requiring UCST behavior. This limitation has recently motivated theoretical and experimental efforts towards the modification of betaine-based monomers and the development of polyzwitterions with an UCST in the physiological range. These studies culminated in the discovery of poly(sulfabetaine) (pzB), which differs structurally from poly(sulfobetaine) (pSB) by bearing a sulfate instead of a sulfonate moiety as the anionic group. ${ }^{38}$ In this way, it is possible to obtain an ion pair of similar charge densities with respect to the original SB monomer (the anionic sulfate group and the cationic trimethyl ammonium group of the $\mathrm{ZB}$ monomer). Yet, this ion pair presents a stronger self-association and a lower interaction with water compared to the sulfonate/trimethyl ammonium ion pair of the SB monomer, in agreement with Collins, "law of matching water affinity". ${ }^{39}$

This recent discovery provides now an attractive opportunity to exploit the unique properties of this polymer and to synthesize biodegradable zwitterionic nanoparticles (NPs) that exhibit an UCST in physiological solutions in a biologically relevant temperature range. This behavior could be exploited for instance in important applications in the context of bioseparation and controlled drug release.

In this work, we develop and describe a strategy to generate this new class of biodegradable zwitterionic NPs by synthesizing poly(sulfobetaine-co-sulfabetaine) ( $\mathrm{p}(\mathrm{SB}-\mathrm{co}-\mathrm{ZB})$ ) copolymers via reversible addition-fragmentation chain transfer (RAFT) polymerization. These $\mathrm{p}(\mathrm{SB}-\mathrm{co}-\mathrm{ZB})$ macromolecular chain transfer agents (macro CTAs) are then chain-extended with biodegradable caprolactone-based macromonomers $\left(\mathrm{HEMACL}_{n}\right.$ ) via RAFT emulsion polymerization. This macromolecular architecture allows us to independently control several crucial properties of the NPs by modifying the individual components of the copolymer. Specifically, we demonstrate the possibility of controlling the value of the UCST by changing the length and the composition of the $\mathrm{p}(\mathrm{SB}-\mathrm{co}-\mathrm{ZB})$ copolymers, the size of the NPs by tuning the length of the hydrophobic portion of the copolymer and the biodegradation rate by modifying the length of the oligoester macromonomer. After achieving the synthesis of biodegradable NPs with a transition temperature close to the body temperature at physiological $\mathrm{NaCl}$ concentration, we show the potential of these materials in the context of drug delivery by controlling the release of a drug-mimic molecule (i.e. pyrene) upon temperature variation.

\section{Experimental section}

\subsection{Materials}

2-Hydroxyethyl methacrylate (HEMA, 97\%, MW = 130.14, Sigma Aldrich), $\varepsilon$-caprolactone (CL, 97\%, MW = 114.14, Sigma Aldrich), stannous octoate ( $\mathrm{Sn}(\mathrm{Oct})_{2}, 92.5-100 \%, \mathrm{MW}=405.12$, Sigma Aldrich), sodium sulfate $\left(\mathrm{Na}_{2} \mathrm{SO}_{4}, \geq 99 \%, \mathrm{MW}=142.04\right.$, Sigma Aldrich), 4,4'-azobis(4-cyanovaleric acid) (ACVA, $\geq 98 \%$, MW $=280.28$, Sigma Aldrich), 4-cyano-4-(phenylcarbonothioylthio)pentanoic acid (CPA, $\geq 97 \%$, MW = 279.38, Sigma Aldrich), [2-(methacryloyloxy)ethyl]dimethyl-(3-sulfopropyl) ammonium hydroxide (SB, 97\%, MW = 279.35, Sigma Aldrich), ethanol ( $\geq 99.8 \%$, MW $=46.07$, Sigma Aldrich), acetonitrile (ACN, 99.99\%, MW = 41.05, Fisher Chemicals), 2-(dimethylamino)ethyl methacrylate (DMAEMA, 98\%, MW $=157.21$, Sigma Aldrich), 1,3-propanediol cyclic sulfate (TMS, 98\%, MW $=138.14$, Sigma Aldrich), dimethyl sulfoxide (DMSO, $\leq 0.02 \%$ water, $\mathrm{MW}=78.13$, Sigma Aldrich), pyrene $(98 \%, \mathrm{MW}=$ 202.25, Sigma Aldrich), and sodium chloride ( $\mathrm{NaCl}, \geq 99.5 \%$, MW $=58.44$, Sigma Aldrich) were used as received. All solvents were of analytical grade purity and used without further treatment.

\subsection{Synthesis of the $\mathrm{ZB}$ monomer}

The ZB monomer was synthesized via an addition reaction according to a procedure previously reported. ${ }^{38}$ Briefly, $5 \mathrm{~g}$ (31.8 mmol) of DMAEMA and $4 \mathrm{~g}(29 \mathrm{mmol})$ of TMS were respectively dissolved in $5 \mathrm{~mL}$ and $35 \mathrm{~mL}$ of acetonitrile at ambient temperature. The solutions were added to a $100 \mathrm{~mL}$ septum-sealed round bottom flask equipped with a magnetic 
stirrer and the reaction was carried out at $50{ }^{\circ} \mathrm{C}$ for three days. At the end of the reaction, the monomer was precipitated by cooling the mixture to ambient temperature and by adding acetone. After filtration and further washing with acetone, the solid was dried in a vacuum oven at $30{ }^{\circ} \mathrm{C}$ to remove all the residual acetone and characterized via proton nuclear magnetic resonance $\left({ }^{1} \mathrm{H}\right.$ NMR) using deuterium oxide $\left(\mathrm{D}_{2} \mathrm{O}\right)$ as the solvent. This analysis confirmed that the monomer was obtained with high purity (see Fig. S1†).

\subsection{Synthesis of polyzwitterions with UCST behaviour}

The polyzwitterionic pSB homopolymer and $\mathrm{p}(\mathrm{SB}-\mathrm{co}-\mathrm{ZB})$ copolymers were synthesized via RAFT polymerization in an ethanol/acetic buffer $(\mathrm{pH}=4.5)(20 / 80 \mathrm{v} / \mathrm{v})$ mixture using ACVA as the initiator, CPA as the chain transfer agent (CTA) and $1 \mathrm{M}$ $\mathrm{NaCl}$ with respect to the acetic buffer volume in the case of $\mathrm{p}(\mathrm{SB}-\mathrm{Co}-\mathrm{ZB})$ for the "salting" effect promotion. The monomer concentration was set equal to $20 \% \mathrm{w} / \mathrm{w}$ and the initiator to CTA molar ratio was set to $1 / 3$. Different monomer to CTA molar ratios (corresponding to different degrees of polymerization, $\mathrm{DP}=200,300,400$ and 600) were adopted for the pSB. For the $\mathrm{p}(\mathrm{SB}-\mathrm{co}-\mathrm{ZB})$, the DP was fixed to 200 and the $\mathrm{ZB}$ molar fraction was varied between 0.25 and 1 . As an example, $\mathrm{p}(200 \mathrm{SB})$ was synthesized by dissolving $16 \mathrm{mg}(57 \mu \mathrm{mol})$ of ACVA, $48 \mathrm{mg}(0.17 \mathrm{mmol})$ of CPA and $9.58 \mathrm{~g}(34 \mathrm{mmol})$ of SB in $40 \mathrm{~mL}$ ethanol/acetic buffer mixture and poured in a $100 \mathrm{~mL}$ septum-sealed round bottom flask equipped with a magnetic stirrer. The mixture composition was adjusted to obtain the different desired copolymers. The mixture was purged for 20 minutes by bubbling nitrogen and then placed in a pre-heated oil bath at $65{ }^{\circ} \mathrm{C}$ under magnetic stirring. The polymerization was carried out for 24 hours and after the completion of the reaction, an aliquot was taken and dried in a vacuum oven to perform ${ }^{1} \mathrm{H}$ NMR $\left(\mathrm{D}_{2} \mathrm{O}\right)$ and gel permeation chromatography (GPC) analysis. The final polymer was purified by a double precipitation in acetone followed by centrifugation to enhance the recovery. It was then dried in a vacuum oven at $35{ }^{\circ} \mathrm{C}$ to remove all the residual acetone and finally stored at $-20^{\circ} \mathrm{C}$.

\subsection{Synthesis of caprolactone-based biodegradable macromonomers}

The lipophilic biodegradable macromonomer was produced via the ring opening polymerization (ROP) of $\varepsilon$-caprolactone using HEMA as the initiator, following a previously reported protocol. ${ }^{40,41}$ The $\mathrm{Sn}(\mathrm{Oct})_{2}$ to HEMA molar ratio was set at 1 / 200 while the monomer to initiator molar ratio was varied from 1 to 3 to 5 . As an example, $\mathrm{HEMACL}_{3}$ was synthesized by weighing $10 \mathrm{mg}$ of $\mathrm{Na}_{2} \mathrm{SO}_{4}$ and $7.9 \mathrm{~g}(69 \mathrm{mmol})$ of CL in a $25 \mathrm{~mL}$ septum-sealed round bottom flask equipped with a magnetic stirrer, which was then placed in an oil bath at $130{ }^{\circ} \mathrm{C}$ under magnetic stirring. Then, $3 \mathrm{~g}$ ( $\left.23 \mathrm{mmol}\right)$ of HEMA and $47 \mathrm{mg}(116 \mu \mathrm{mol})$ of $\mathrm{Sn}(\mathrm{Oct})_{2}$ were mixed in a $10 \mathrm{~mL}$ glass vial and transferred to the pre-heated flask with a syringe. After 2.5 hours, the reaction was stopped and an aliquot was taken to perform ${ }^{1} \mathrm{H}$ NMR $\left(\mathrm{CDCl}_{3}\right)$ and GPC analyses.

\subsection{Synthesis of zwitterionic biodegradable NPs with an UCST behavior}

Amphiphilic block copolymers assembled into NPs with an UCST were synthesized via RAFT emulsion polymerization by chain-extending the $\mathrm{p}(\mathrm{SB}-\mathrm{co}-\mathrm{ZB})$ macromolecular CTA (macro CTA) with HEMACL ${ }_{n}$. The reaction was performed in a 20/80 v/v mixture of ethanol/acetic buffer $(\mathrm{pH}=4.5)$, using ACVA as the initiator. The DP of the lipophilic block was set to 20,40 or 60 . Considering the $\mathrm{p}(150 \mathrm{SB}-50 \mathrm{ZB})-b-\mathrm{p}$ $\left(20 \mathrm{HEMACL}_{3}\right)$ as an example, $0.37 \mathrm{mg}(1.3 \mu \mathrm{mol})$ of ACVA, $38 \mathrm{mg}(80 \mu \mathrm{mol})$ of $\mathrm{HEMACL}_{3}, 227 \mathrm{mg}(\sim 4 \mu \mathrm{mol})$ of $\mathrm{p}(150 \mathrm{SB}-50 \mathrm{ZB})$ and $0.28 \mathrm{~g}$ of $\mathrm{NaCl}$ were dissolved in $10 \mathrm{~mL}$ ethanol/acetic buffer mixture and poured in a $50 \mathrm{~mL}$ septum-sealed round bottom flask equipped with a magnetic stirrer. The mixture was purged with nitrogen for 15 minutes and placed in a pre-heated oil bath at $65{ }^{\circ} \mathrm{C}$ under magnetic stirring. The polymerization was carried out for 24 hours; the final product was dialyzed against $0.9 \%$ $\mathrm{NaCl}$ aqueous solution for one day to bring the suspension to physiological conditions and the cloud points were detected via Dynamic Light Scattering (DLS).

\subsection{Degradation test}

The degradation kinetics of the thermo-responsive polymers was analyzed at $37^{\circ} \mathrm{C}$ and $\mathrm{pH}=14$ via dynamic light scattering, monitoring the relative increase of the scattered intensity with respect to time zero and the average NP size. The NP dispersion was diluted to $0.3 \% \mathrm{w} / \mathrm{w}$ in physiological solution and $1.5 \mathrm{~mL}$ of the dispersion were mixed with $1 \mathrm{~mL}$ of $0.1 \mathrm{M} \mathrm{NaOH}$ solution before starting the analysis.

\subsection{Pyrene loading and release}

Pyrene-loaded NPs were prepared via the "syringe method"42 by dissolving pyrene in DMSO $(0.25 \mathrm{mg}$ in $50 \mu \mathrm{L})$ and setting the pyrene to polymer mass ratio at $0.25 \mathrm{mg} / 20 \mathrm{mg}$. The loading temperature was chosen as $45{ }^{\circ} \mathrm{C}$. Therefore, the pyrene solution, the NP suspension and the instruments needed for the loading (syringe and needle) were left to equilibrate at the specific test temperature before usage. The pyrene solution was then aspired using a syringe pre-filled with $4 \mathrm{~mL}$ of the NP suspension. The mixture was ejected and aspired three times to achieve sufficient turbulent conditions to lead to NP swelling and pyrene loading. To determine the amount of loaded pyrene, the polymer was precipitated by adding $1.5 \mathrm{~mL}$ of the loaded NP suspension in a vial and incubating it at $4{ }^{\circ} \mathrm{C}$. The liquid supernatant was removed after centrifugation and freeze-dried and the residual pyrene was dissolved in $4 \mathrm{~mL}$ of acetonitrile. The samples were vortex-stirred for $30 \mathrm{~s}$ and centrifuged at $5000 \mathrm{rpm}$ for 5 minutes and the recovered supernatant was placed in a glass cuvette and analyzed via UV-Vis spectrometry. To determine the pyrene concentration from absorbance data, a calibration curve was constructed by analyzing pyrene solutions in acetonitrile with concentrations ranging from 1 to $1000 \mathrm{mg}$ $\mathrm{L}^{-1}$. The loading efficiency, i.e. the percentage of pyrene effec-
1 
tively entrapped within the polymeric NPs, was calculated according to eqn (1).

$$
\%_{\mathrm{PYR}, \mathrm{LOADED}}=100 \times\left(1-\frac{m_{\mathrm{PYR}, \mathrm{NL}}}{m_{\mathrm{PYR}, 0}}\right)
$$

where $\%_{\text {PYR,LOADED }}$ is the loading efficiency, $m_{\mathrm{PYR}, 0}$ is the mass of added pyrene and $m_{\mathrm{PYR}, \mathrm{NL}}$ is the mass of pyrene not loaded, i.e. recovered in the supernatant.

The release profiles for the pyrene-loaded $\mathrm{p}(110 \mathrm{SB}-90 \mathrm{ZB})-b$ $\mathrm{p}\left(20 \mathrm{HEMACL}_{3}\right)$ NPs were evaluated via UV-Vis spectroscopy according to two different procedures at 30 and $45{ }^{\circ} \mathrm{C}$ (i.e. below and above the $T_{\mathrm{cp}}$, respectively). For the latter, $3 \mathrm{~mL}$ of loaded NP suspension were added to a dialysis membrane (Slide-A-Lyzer dialysis cassette, Thermo Scientific, molecular weight cut-off $3.5 \mathrm{kDa}$ ) and dialyzed against $500 \mathrm{~mL}$ of physiological solution kept at the same temperature. Aliquots (i.e. $0.1 \mathrm{~mL}$ ) were taken from the membrane at predetermined times and freeze-dried and the pyrene was solubilized in $1 \mathrm{~mL}$ of acetonitrile before UV-Vis analysis. The release profile was evaluated according to eqn (2).

$$
\text { Released }_{\mathrm{PYR}}(t)=100 \times\left(1-\frac{m_{\mathrm{PYR}}}{m_{\mathrm{PYR}, 0 \mathrm{~h}}}\right)
$$

where Released $\mathrm{PYR}_{\mathrm{PY}}(t)$ is the percentage of pyrene released at the specific time $t$ and $m_{\mathrm{PYR}}$ and $m_{\mathrm{PYR}, 0 \mathrm{~h}}$ are the amounts of pyrene in the membrane at time $t$ and time zero, respectively.

For the experiment at $30^{\circ} \mathrm{C}$ (i.e. below the $T_{\mathrm{cp}}$ ), the polymer precipitates due to the formation of microaggregates at the specific test temperature. Therefore, to quantify the pyrene release as a function of time, $1.5 \mathrm{~mL}$ of the loaded NP suspension were added to a vial pre-heated to $30^{\circ} \mathrm{C}$, the polymer was left to precipitate and the supernatant was removed by pipetting. $3 \mathrm{~mL}$ of physiological solution were carefully added to the vial, removed at predetermined times and replaced by fresh solution. The withdrawn solutions were freeze-dried and pyrene was dissolved in $1 \mathrm{~mL}$ of acetonitrile for its quantification via UV-Vis analysis. The release profile was evaluated according to eqn (3):

$$
\text { Released }_{\mathrm{PYR}}\left(t_{n}\right)=\text { Released }_{\mathrm{PYR}}\left(t_{n-1}\right)+100 \times \frac{m_{\mathrm{PYR}}\left(t_{n}\right)}{m_{\mathrm{PYR}, 0 \mathrm{~h}}}
$$

where Released ${ }_{\mathrm{PYR}}\left(t_{n}\right)$ is the percentage of pyrene released at the time point $t_{n}$, Released PYR $_{\text {P }}\left(t_{n-1}\right)$ is the percentage of released pyrene at the time point $t_{n-1}, m_{\mathrm{PYR}}\left(t_{n}\right)$ is the mass of pyrene in the supernatant recovered at the time point $t_{n}$ and $m_{\mathrm{PYR}, 0 \mathrm{~h}}$ is the mass of pyrene at time zero. Three independent experiments were performed at each temperature.

\subsection{Characterization}

${ }^{1} \mathrm{H}$ NMR spectra were recorded on a Bruker $400 \mathrm{MHz}$ spectrometer and the samples were prepared by dissolving $20 \mathrm{mg}$ of polymer in $0.7 \mathrm{~mL}$ of a deuterated solvent (specified for each polymer in the reaction methods).

Gel Permeation Chromatography (GPC) was performed on Jasco AS-2055 Plus apparatus. For the organic separation, samples were dissolved at $5 \mathrm{mg} \mathrm{mL}{ }^{-1}$ in THF and filtered through a $0.45 \mu \mathrm{m}$ pore-size PTFE membrane. The separation was performed at a flow rate of $0.5 \mathrm{~mL} \mathrm{~min}{ }^{-1}$, at $35^{\circ} \mathrm{C}$, with three different Superchrom PL gel $5 \mu \mathrm{m}$ columns (Polymer laboratories Ltd, UK; two columns had pore sizes of the Mixed-C type and one was an Oligopore; $300 \mathrm{~mm}$ length and $7.5 \mathrm{~mm} \mathrm{ID)}$ ). Polystyrene standards were employed for the construction of the calibration curve. For the aqueous separation, samples were dissolved at $5 \mathrm{mg} \mathrm{mL} \mathrm{mL}^{-1}$ in $0.05 \mathrm{M} \mathrm{Na}_{2} \mathrm{SO}_{4} /$ acetonitrile $(80 / 20 \mathrm{v} / \mathrm{v})$ solution and filtered through a $0.45 \mu \mathrm{m}$ pore-size nylon membrane. The separation was performed at a flow rate of $0.5 \mathrm{~mL}$ $\min ^{-1}$, at $35{ }^{\circ} \mathrm{C}$ with a guard and three Suprema columns (Polymer Standards Service; particle size $10 \mathrm{~mm}$ and pore sizes of 100,1000 , and $3000 \AA$ ), and polyethylene glycol standards were employed for the construction of the calibration curve.

Dynamic light scattering analysis was performed on a Zetasizer Nano ZS (Malvern Instrument) at a scattering angle of $173^{\circ}$. For measurements involving a temperature trend, the temperature was increased at intervals of $2{ }^{\circ} \mathrm{C}$, allowing the sample to equilibrate for 10 minutes at each temperature before acquiring the measurement. The results shown in this work correspond to the average of three independent measurements.

UV-Vis measurements were performed on a Jasco V-630 spectrophotometer. The absorbance spectra were recorded in a range from 350 to $280 \mathrm{~nm}$ with an emission width equal to $1.5 \mathrm{~nm}$.

Fluorescence measurements were performed on a Jasco FP-8500 spectrofluorometer with a continuous output Xe arc lamp with shielded lamp housing $(150 \mathrm{~W})$. Excitation was carried out at $335 \mathrm{~nm}$. The emission spectra were recorded in a range from 350 to $450 \mathrm{~nm}$. Excitation and emission band widths were set at 5 and $2 \mathrm{~nm}$, respectively. From the pyrene emission spectra, the intensity ratio $\left(I_{3} / I_{1}\right)$ of the third band $(384 \mathrm{~nm})$ to the first band $(373 \mathrm{~nm})$, which is a sensitive parameter characterizing the polarity of the probe's environment, ${ }^{43}$ was analyzed.

Imaging of the $\mathrm{p}(\mathrm{SB}-\mathrm{co}-\mathrm{ZB})$ copolymer and NP droplets in PBS and cell lysates was performed using a $60 \times$ oil objective (CFI Plan Apo Lambda, Nikon) on an epi-fluorescence microscope (Eclipse Ti-E, Nikon) equipped with an Omicron LedHub light engine. The data acquisition was performed using a 384-well plate (MatriPlate, Glass Bottom, Brooks). Cell lysates were produced from $E$. Coli BL21 Gold (DE3) competent cells via sonication in PBS and centrifugation.

Transmission electron microscopy (TEM) was performed on a Hitachi HT 7700 (ScopeM, ETH Zurich). The nanoparticles were diluted ten times in $180 \mathrm{~g} \mathrm{~L}^{-1} \mathrm{NaCl}$ solution. $5 \mu \mathrm{L}$ of NP suspension were dropped onto a 400 mesh carbon-coated copper grid, and stained with $5 \mu \mathrm{L}$ of $0.4 \% \mathrm{w} / \mathrm{w}$ uranyl acetate solution. The micrographs were acquired with a magnification of $50.0 \mathrm{k} \times$ and at an acceleration voltage of $100 \mathrm{kV}$. The NP size distribution was reconstructed from 6 TEM images using ImageJ and statistical analysis was performed with Origin 8.

Elemental analysis via Energy Dispersive X-ray Spectroscopy (EDXS) was performed on an FEI Talos F200X (ScopeM, ETH Zurich). The nanoparticles were not stained for EDXS analysis. 


\section{Results and discussion}

\subsection{Influence of the SB and ZB composition on the UCST behavior at different ionic strengths}

First, we synthesized pSB and pZB homopolymers by RAFT polymerization of the $\mathrm{SB}$ and $\mathrm{ZB}$ monomers, respectively. The $\mathrm{p}(\mathrm{ZB})$ homopolymers exhibited an UCST $>100{ }^{\circ} \mathrm{C}$ (Table S1†). In contrast, the pSB homopolymers presented a clear phase separation in the range between 19 and $32{ }^{\circ} \mathrm{C}$ in pure water (Fig. S2, Table S1 $\dagger$ ). Within this range, the cloud point varied as a linear function of the degree of polymerization and therefore of the polymer molecular weight. However, independently of the polymer molecular weight, the phase separation disappeared under physiological saline conditions (i.e. $0.9 \% \mathrm{w} / \mathrm{w}$ $\mathrm{NaCl}$ solution).

In contrast, when SB was copolymerized with the $\mathrm{ZB}$ monomer, the produced zwitterionic copolymers (structure in Fig. 1a and ${ }^{1} \mathrm{H}$ NMR spectrum in Fig. S3 $\dagger$ ) retained the UCST behavior in a biologically relevant temperature range $\left(30-45^{\circ} \mathrm{C}\right)$ at physiological $\mathrm{NaCl}$ concentration ${ }^{38}$ (Fig. 1b). We note that the use of the RAFT copolymerization in the synthesis of p(SB-coZB) was important to generate narrowly dispersed chains (Table $\mathrm{S} 1 \dagger$ ) with a reduced interchain composition drift and hence with a sharp and well-defined phase separation. ${ }^{44}$

The phase separation of these $\mathrm{p}(\mathrm{SB}-\mathrm{co}-\mathrm{ZB})$ copolymers upon cooling resulted in the formation of liquid droplets (Fig. 1c) that coalesced until the formation of a homogeneous polymerrich liquid phase (Movie $\mathrm{S} 1 \dagger$ ). These liquid droplets are composed of polymer chains held together by strong electrostatic interactions. Importantly, we observed that such interactions are specific and allow retaining the liquid-liquid phase separation even in a complex fluid such as an E. Coli cell lysate containing many different proteins and cell debris (Fig. 1d).

In addition, in contrast to other UCST-based materials, such as poly( $N$-acryloyl glycinamide) (PNAGA) ${ }^{24}$ ureido derivatized polymers, ${ }^{26}$ and poly(acrylamide-co-acrylonitrile) copolymers, ${ }^{20}$ the electrostatic interactions in the zwitterionic polymers promote the formation of a hydration layer round the polymer-rich droplets, which minimizes the adsorption of macromolecules from the solution. ${ }^{45}$ This high hydrophilicity makes these materials a promising choice for the fabrication of the shell of micelles or NPs intended for drug delivery applications in which undesired immunological responses and the accelerated blood clearance effect must be avoided. ${ }^{46,47}$ Indeed, we verified that the green fluorescent protein (GFP), used as a model protein, did not diffuse into the polymer-rich phase of our materials (Fig. 1e).

\subsection{Zwitterionic biodegradable NPs with UCST behavior}

We next generated UCST-type biodegradable NPs from the linear zwitterionic $\mathrm{p}$ (SB-co-ZB) copolymers described above. For this purpose, we chain-extended the previously synthesized zwitterionic $\mathrm{p}$ (SB-co-ZB) macro chain transfer agents (macro CTA) via RAFT emulsion polymerization with biodegradable HEMACL $_{n}$ macromonomers produced via ROP, with $n$ ranging from 0 to 5 (Table $\mathrm{S} 2 \dagger$ and ${ }^{1} \mathrm{H}$ NMR spectrum in Fig. S4 $\dagger$ ). With this method, we obtained spherical NPs (Fig. 2a) that exhibit a relatively narrow particle size distribution as evaluated by TEM (Fig. S5 and S6†) and average hydrodynamic diameters in the range 60-190 $\mathrm{nm}$. These NPs are composed of well-defined block copolymers. ${ }^{48}$ In particular, the NP core is composed of biodegradable PCL-based lipophilic lateral chains while the zwitterionic UCST portion is confined on the NP surface, providing colloidal stability (see elemental analysis via EDXS, Fig. S7†).

The UCST behavior of these NPs above and below their binodal curve is schematized in Fig. 2b. At temperature above the $T_{\mathrm{cp}}$, the $\mathrm{p}(\mathrm{SB}-\mathrm{co}-\mathrm{ZB})$ segments are extended in the water phase and stabilize the lipophilic NP core. Under these conditions, we expect narrowly distributed NPs. In contrast, at temperature below the $T_{\mathrm{cp}}, \mathrm{p}(\mathrm{SB}-\mathrm{co}-\mathrm{ZB})$ chains collapse on the NP surface and induce phase separation of the zwitterionic NP corona, thereby leading to NP coagulation into micrometric aggregates. To verify this behavior, the phase separation of the $\mathrm{p}(110 \mathrm{SB}-90 \mathrm{ZB})-b-\mathrm{p}\left(20 \mathrm{HEMACL}_{3}\right)$ based NPs in physiological solution was tracked via dynamic light scattering (DLS) by recording the NP size (Fig. 2c) and relative scattering intensity (Fig. S8 $\dagger$ ) as a function of temperature. At $40{ }^{\circ} \mathrm{C}$, slightly above the typical body temperature, stable NPs with a low polydispersity index $(\mathrm{PDI}<0.15)$ were obtained and no aggregates were observed by optical microscopy (top panel in Fig. 2b). Upon cooling, the NP size abruptly increases from $90 \mathrm{~nm}$ to $1.8 \mu \mathrm{m}$ while the scattering intensity decreases, indicating the formation of large NP clusters that sediment over time, as confirmed also by optical microscopy (bottom panel in Fig. 2b, Movie S2 $\dagger$ ). The $T_{\text {cp }}$ of the NPs can be evaluated as the inflection point of the size curve as a function of temperature and, in this case, was equal to the physiological temperature of $37{ }^{\circ} \mathrm{C}$ (Fig. 2c). Overall, these results confirm that the NPs inherited the UCST behavior of the zwitterionic blocks under physiological conditions.

The characteristic comb-like structure of the block copolymers constituting the NPs allows us to independently control the main properties of the NPs. As schematically depicted in Fig. 3a, the composition of the zwitterionic portions controls the UCST behavior of the NPs, while the number ( $m$, Fig. 2a) and the length (n, Fig. 2a) of the hydrophobic lateral chains control the NP size and degradation rate, respectively.

In particular, the NP $T_{\mathrm{cp}}$ can be modulated by changing the relative $\mathrm{ZB} / \mathrm{SB}$ feed during the synthesis of the zwitterionic portion of the constituting block copolymers (Fig. 3b). Specifically, the $T_{\mathrm{cp}}$ of the NPs was found to be a linear function of the ZB mole fraction, in analogy to the behavior observed for the linear zwitterionic block (Fig. 1b).

The NP size can be controlled by the length of the lipophilic portion of the block copolymers. As reported in Fig. 3c, the NP size linearly increases with the number of the hydrophobic lateral chains, in agreement with that already shown for similar non-thermo-responsive biodegradable NPs. ${ }^{48}$ The addition of the lipophilic block causes an increase in the $T_{\mathrm{cp}}$ compared to the values found for the zwitterionic block alone (see Fig. 3b and Table $\mathrm{S} 1 \dagger$ ). Interestingly, this increase in the $T_{\mathrm{cp}}$ is not proportional to the number of the oligoester lateral 
(a)<smiles>C=C(C)C(=O)OCC[N+](C)(C)CCC[Si](O)(O)O</smiles>

Sulfobetaine Methacrylate (SB)<smiles>CC(=O)C(=O)OCC[N+](C)(C)CCCOS(O)(O)O</smiles>
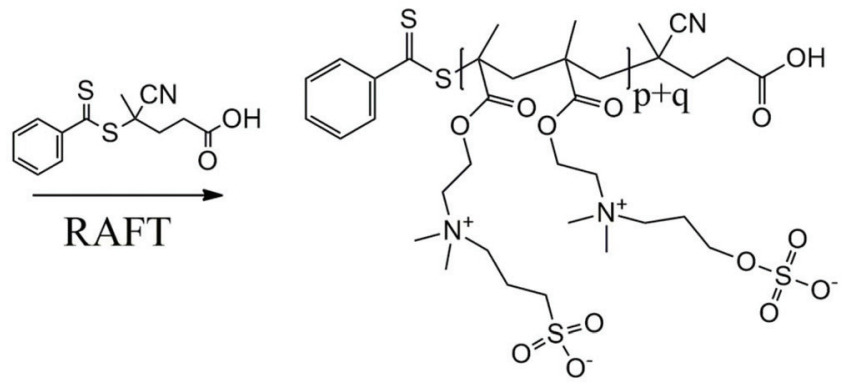

1

\section{Sulf abetaine Methacrylate (ZB)}

(b)

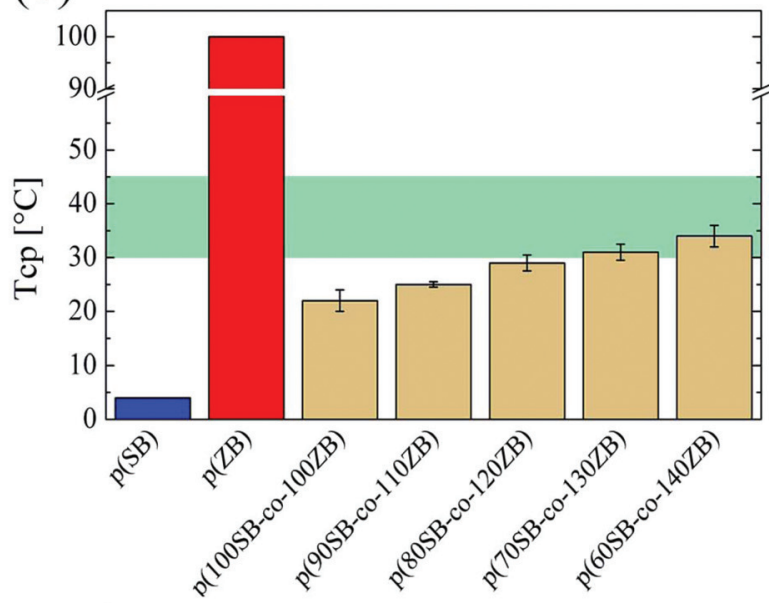

(d)

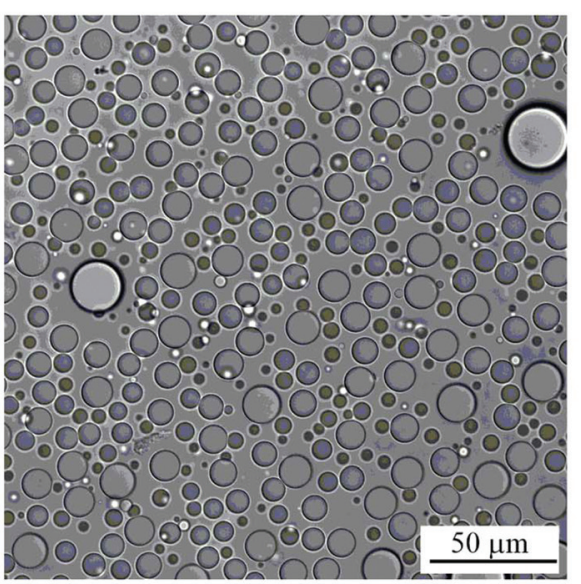

(c)

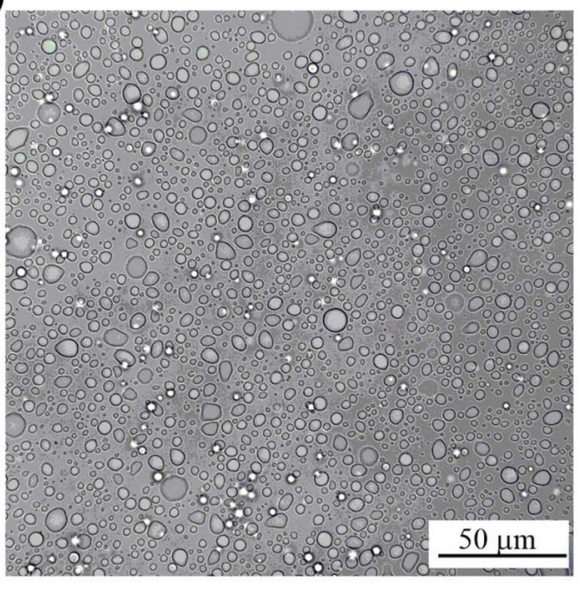

(e)

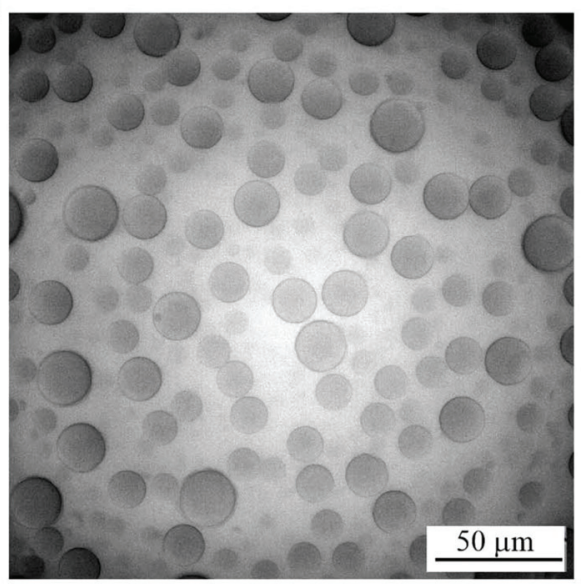

Fig. 1 (a) Structure of the zwitterionic p(SB-co-ZB) copolymers obtained via RAFT polymerization. (b) $T_{\mathrm{cp}}$ of $5 \%$ w/w copolymer solutions in a physiological $(0.9 \% \mathrm{w} / \mathrm{w} \mathrm{NaCl})$ medium. (c) Optical micrograph of $\mathrm{p}(60 \mathrm{SB}-\mathrm{co}-140 \mathrm{ZB})$ recorded at $25^{\circ} \mathrm{C}$ in a physiological medium. (d) Optical micrograph of $2.5 \mathrm{mg} \mathrm{mL}^{-1} \mathrm{p}(60 \mathrm{SB}-\mathrm{co}-140 \mathrm{ZB})$ copolymers in $E$. Coli cell lysates at $25^{\circ} \mathrm{C}$. (e) Optical micrograph of $5 \mathrm{mg} \mathrm{mL}^{-1} \mathrm{p}(60 \mathrm{SB}-\mathrm{co}-140 Z \mathrm{~B}) \mathrm{copoly}$ mers in the presence of $1 \mu \mathrm{M}$ GFP in PBS at $25^{\circ} \mathrm{C}$. The GFP fluorescence is localized outside the droplets, which appear dark.

chains, as can be seen in Fig. 3d. In fact, the cloud point remains almost constant when $m$ is in the range 20-60 units. It is worth noticing that this behavior can also be seen when LCST-type chains are used to stabilize NPs with a similar lipophilic core. ${ }^{49}$ In this way, it is possible to synthesize NPs of different sizes sharing the same $T_{\mathrm{cp}}$ by varying the number of the lipophilic chains and keeping constant the composition of the zwitterionic UCST portion.
Subsequently, we have also studied the impact of the length $(n)$ of the hydrophobic lateral chains on the NP properties and degradation time. In contrast to that previously seen for the number of lateral chains $(m)$, we observed an increase in the NP cloud point by increasing $n$ (Fig. 3e). This feature can be used to independently tune the NP cloud point and the NP size by changing $n$ and $m$, respectively, using the same zwitterionic block. The impact of $n$ is, however, more important for 
(a)

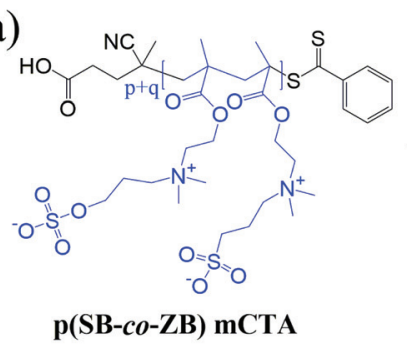

(b)

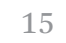
considered as the inflection point of the curve.

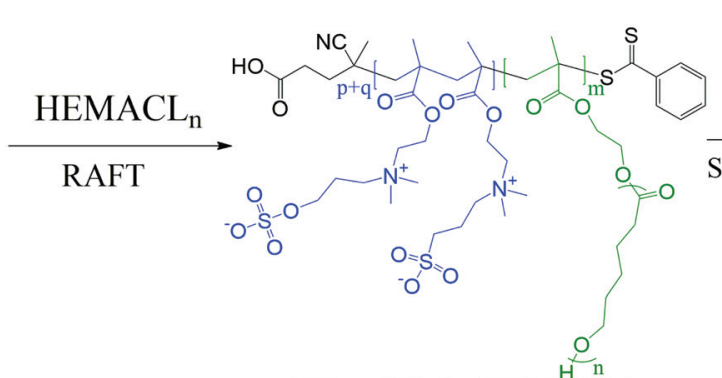

p(SB-co-ZB)- $b$-p $\left(\right.$ HEMĂCL $\left._{\mathrm{n}}\right)$

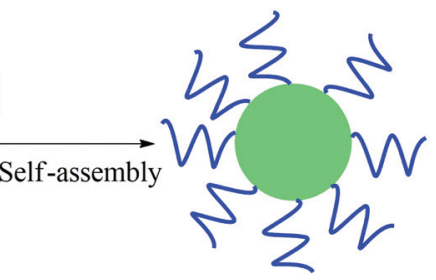

(c)
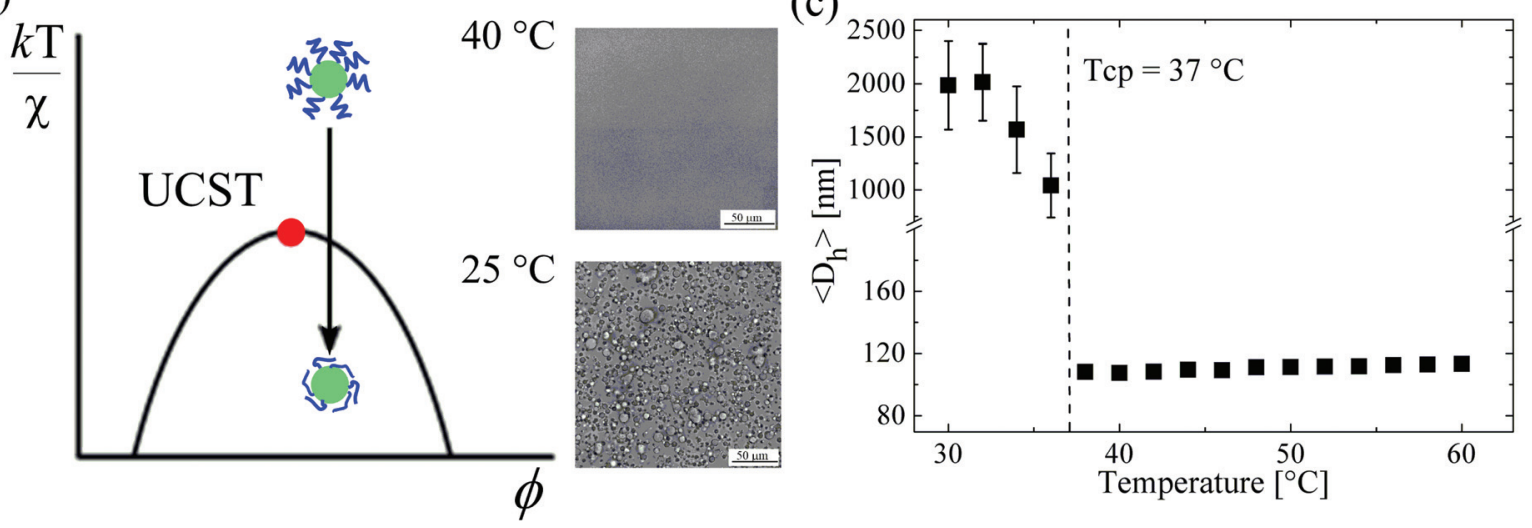

10

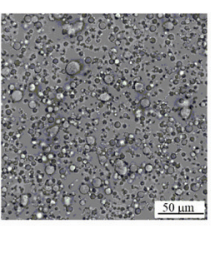

Fig. 2 (a) Schematic of the synthesis route and composition of the block copolymers $p(S B-c o-Z B)-b-p\left(H E M A C L_{n}\right)$ produced via RAFT emulsion polymerization starting from the $\mathrm{p}(\mathrm{SB}-\mathrm{CO}-\mathrm{ZB})$ macro CTAs. (b) Phase diagram of the produced NPs with UCST behavior in the physiological temperature range. The insets show optical micrographs of the $\mathrm{p}(110 \mathrm{SB}-90 \mathrm{ZB})-b-\mathrm{p}\left(20 \mathrm{HEMACL}_{3}\right) \mathrm{NP}$ dispersion $(5 \% \mathrm{w} / \mathrm{w})$ in $0.9 \% \mathrm{w} / \mathrm{w} \mathrm{NaCl}$ solution at $40{ }^{\circ} \mathrm{C}$ (top panel) and $25^{\circ} \mathrm{C}$ (bottom panel). (c) Average hydrodynamic diameter for the same NP dispersion as a function of the temperature. The $T_{\mathrm{cp}}$ was

the specific modulation of the NP degradation time. In fact, the hydrophobic lateral chains are prone to hydrolysis due to the presence of ester bonds ${ }^{36}$ and the length and number of these lateral chains strongly affect the degradation time of the NPs.

To measure the degradation rate of the supramolecular NP aggregates, we tracked their size and the relative scattering intensity during incubation at body temperature under accelerated degradation conditions $(\mathrm{pH}=14$ (ref. 48)) (Fig. S9†). In a first phase (from 0 to 8 hours), the scattered light decreased, indicating the degradation of the NP aggregates due to the hydrolysis of the lateral caprolactone chains. In addition to the degradation of the aggregates, this hydrolysis decreases the length of the lateral chains, which, in turn, affects the $T_{\mathrm{cp}}$ of the NPs (Fig. 3e). After about 8 hours, the breakage of the lateral caprolactone chains led to a shift of the $T_{\mathrm{cp}}$ of the system below body temperature, leading to the formation of a homogeneous NP dispersion. This release of primary particles upon degradation may be in turn responsible for the increase in the scattered light intensity (Fig. S9†). The degradation time was computed as the time required to induce the release of the primary NPs, which is expected to increase with the length of the biodegradable lateral chain, as verified in our experiments (Fig. 3f).

After demonstrating the possibility of producing tunable UCST-type thermo-responsive biodegradable NPs, we tested their ability to load and release a drug mimic molecule (i.e. pyrene) above and below the cloud point (Fig. 4). The pyrene was loaded in the $\mathrm{p}(110 \mathrm{SB}-90 \mathrm{ZB})-b-\mathrm{p}\left(20 \mathrm{HEMACL}_{3}\right)$ NPs at $45{ }^{\circ} \mathrm{C}$, achieving a loading efficiency of $98.21 \pm 1.44 \%$, and the pyrene release was then evaluated below and above the $T_{\mathrm{cp}}$ at 30 and $45{ }^{\circ} \mathrm{C}$, respectively.

At $45{ }^{\circ} \mathrm{C}$, we observed a fast release of pyrene from the NPs, which was largely driven by diffusion and reached $80 \%$ of the initial encapsulated value after 5 hours. In contrast, when the NPs formed a coagulated phase below the $T_{\mathrm{cp}}$, the pyrene was retained in the NPs for a longer period of time, with only $10 \%$ of the initial amount released after 24 hours (Fig. 4a).

The release of pyrene was further monitored by recording the emission spectrum of the molecule, which is an indicator of the polarity of the surrounding environment. Below the $T_{\mathrm{cp}}$, the ratio of the intensities of the third band to the first band in the pyrene emission spectrum $\left(\right.$ i.e. $\left.I_{3} / I_{1}\right)$ was $0.609 \pm 0.007$, which is indicative of pyrene confinement in a non-polar environment and therefore confirms its retention in the lipophilic NP aggregates. In contrast, at $45^{\circ} \mathrm{C}$ this value decreased to $0.590 \pm 0.003$, indicative of the release of pyrene into the polar water phase (Table S3, Fig. S10†).

Our experiment demonstrates the possibility of controlling the release of molecules by changing the phase state of the NPs. Indeed, while the fast release of pyrene above the $T_{\mathrm{cp}}$ impeded the accurate control of the concentration in solution, 
(a)

(c)

(e)
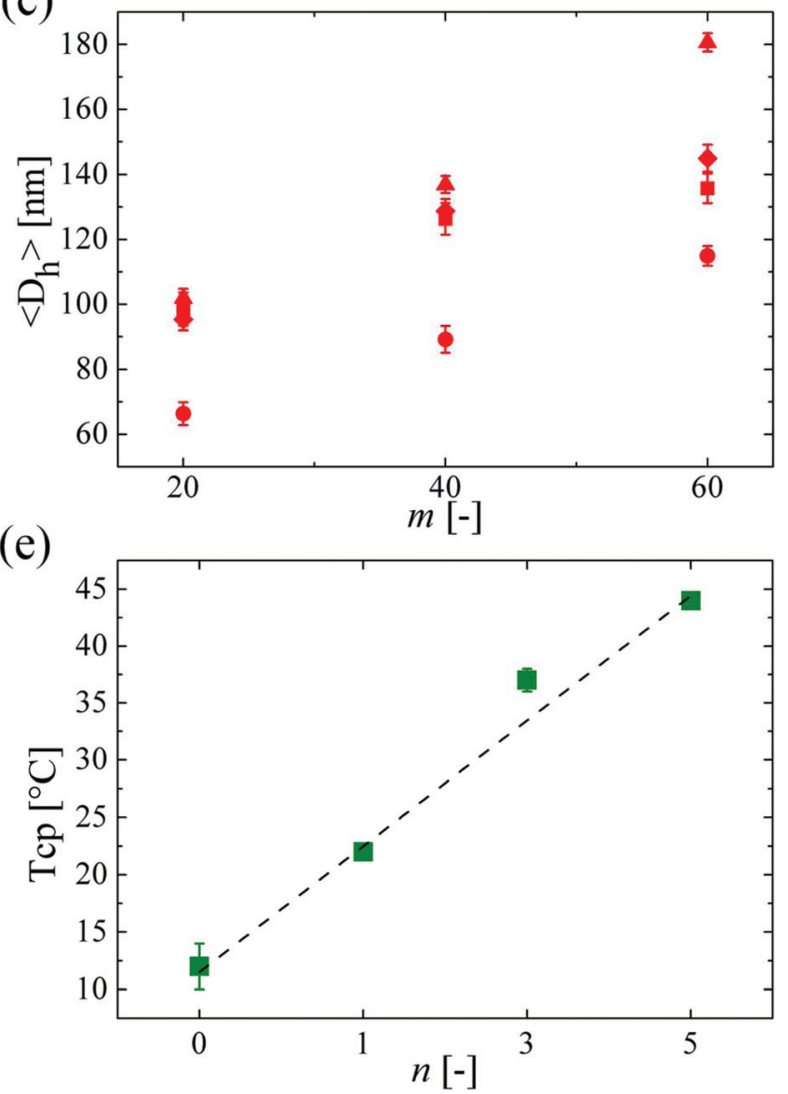

(b)

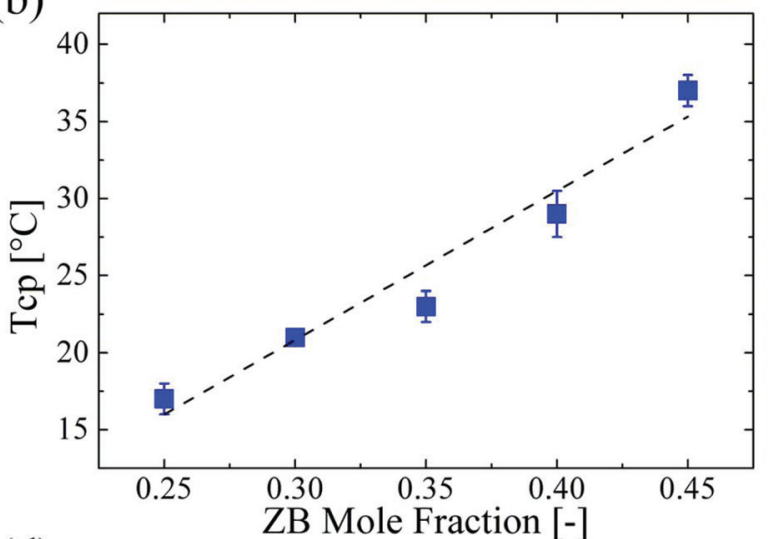

(d)

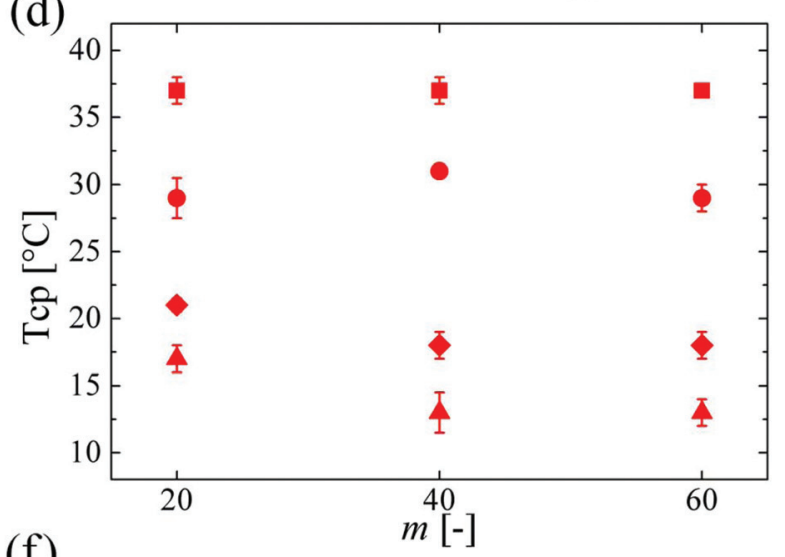

(f)

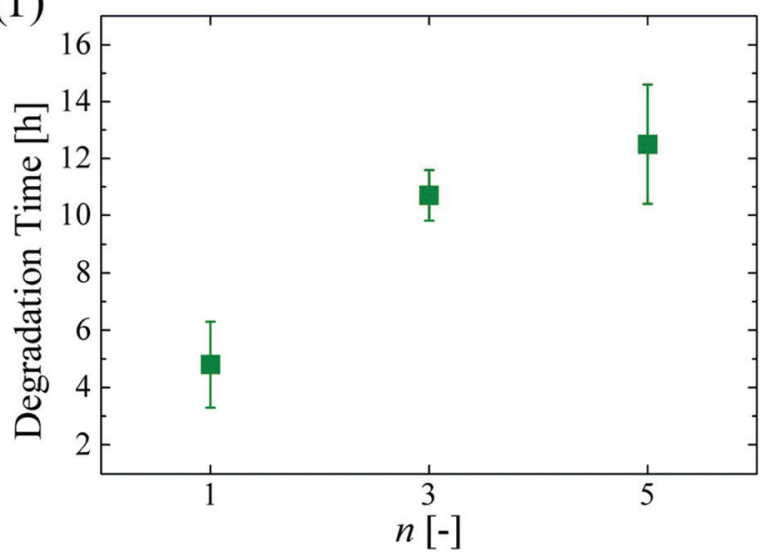

1

below the $T_{\mathrm{cp}}$ we maintained a constant drug concentration within a selected window for a prolonged time of 20 hours (Fig. 4a).

This strategy can pave the way for pharmacological treatment with a reduced number of injections, improved patient compliance and reduced risk of exceeding the safe drug concentration. ${ }^{50}$ For instance, we envision potential applications in the context of novel injectable systems for the local delivery of therapeutics (Fig. 4b). The NP solution could be stored in the coagulated phase below the $T_{\mathrm{cp}}$ and then it could be pre-heated above the human body temperature shortly before injection, thereby allowing administration as a low-viscosity NP suspension. Immediately after injection, the temperature would drop below the $T_{\mathrm{cp}}$, inducing the formation of supramolecular NP aggre- 
(a)

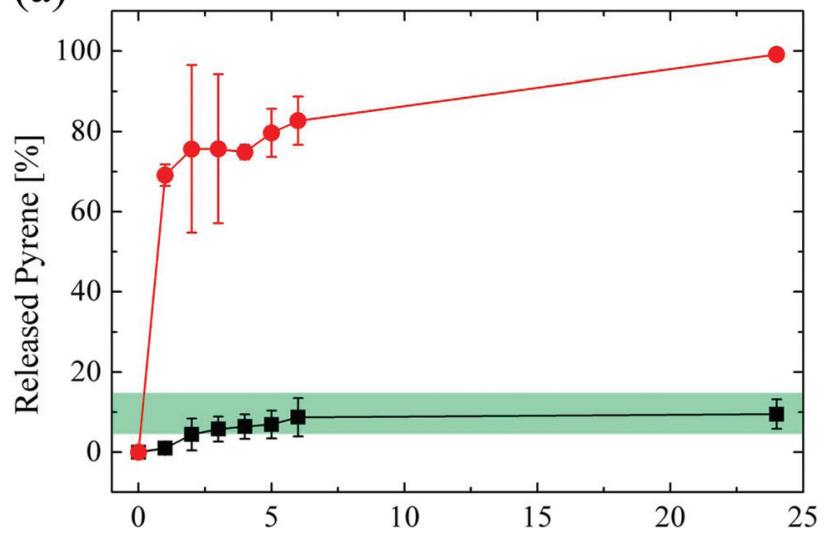

Time $[\mathrm{h}]$

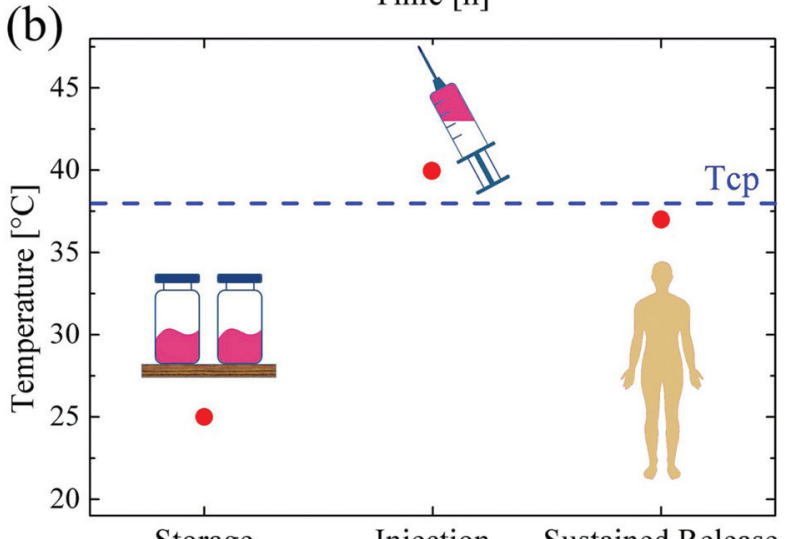

Fig. 4 (a) Pyrene release profiles as a function of time for $\mathrm{p}(110 \mathrm{SB}-90 \mathrm{ZB})-b-\mathrm{p}(2 \mathrm{OHEMACL})$ at $30{ }^{\circ} \mathrm{C}(\mathbf{\square})$ and $45^{\circ} \mathrm{C}(0)$ measured by UV-Vis. The hypothetical therapeutic window is highlighted as a green band. (b) Proposed strategy for storage, administration, and sustained release of the drug for the developed UCST formulation.

gates in situ. This polymer-rich phase can provide a controlled release of the loaded therapeutic, with the additional advantage of the degradation of the drug carrier and its elimination from the body, leading to a low risk of possible toxic bioaccumulation. In fact, the degradation of the lateral oligoester chains that constitute the NP core leaves hydrosoluble chains with a molecular weight lower than the threshold for renal excretion. ${ }^{50}$ Additionally, with respect to more traditional PEG-functionalized NPs, ${ }^{51}$ the zwitterionic chemistry of the system is expected to reduce side effects related to the body immune response and possible allergic reactions. ${ }^{46,47}$

Overall, these results demonstrated the possibility of producing highly tunable biodegradable zwitterionic nanoparticles with an UCST-type phase separation under physiological conditions that can be used to load and modulate the release of a drug within a desired window from few minutes up to 20 hours.

\section{Conclusions}

In this work, we have synthesized for the first time biodegradable zwitterionic NPs that present an UCST-type phase separation in physiological solution in a temperature range relevant for biomedical applications $\left(30<T<45{ }^{\circ} \mathrm{C}\right)$.

These NPs are structurally composed of well-defined block copolymers obtained via the combination of ROP and RAFT polymerization. We have demonstrated that this modular macromolecular architecture opens the possibility of independently tuning several properties of the NPs simply by modulating the stoichiometry of the polymerization reactions. Specifically, we can modify the UCST-type behavior, the NP size, and the aggregate degradation rate by the SB/ZB mole ratio of the zwitterionic portion and on the number and the length of the lipophilic oligoester lateral chains, respectively.

We have shown that the ability to control the phase separation of the NPs via a temperature switch allows us to modulate the release of a drug mimic molecule in a broad interval of time from few minutes to 20 hours. In particular, the rate of release can be drastically decreased by inducing the formation of a polymer-rich phase below the cloud point.

Our NPs with UCST behavior under physiological conditions can pave the way for controlled delivery strategies that are compatible with conventional parenteral administration routes and maintain the concentration of drugs within the desired therapeutic windows for periods of time of several hours, decreasing the number of injections and improving patient compliance.

\section{Conflicts of interest}

The authors declare no conflicts of interest.

\section{Acknowledgements}

We gratefully acknowledge Dr Frank Krumeich of the ScopeM (ETH Zurich) for the elemental analysis via EDXS. This work has been supported by ETH Zurich.

\section{References}

1 S. Strandman and X. X. Zhu, Prog. Polym. Sci., 2015, 42, 154-176.

2 Y. Zuo, Z. Jiao, L. Ma, P. Song, R. Wang and Y. Xiong, Polymer, 2016, 98, 287-293.

3 M. Boustta, P. Colombo, S. Lenglet, S. Poujol and M. Vert, J. Controlled Release, 2014, 174, 1-6.

4 M. Mertoglu, S. Garnier, A. Laschewsky, K. Skrabania and J. Storsberg, Polymer, 2005, 46, 7726-7740.

5 Z. M. O. Rzaev, S. Dinçer and E. Pişkin, Prog. Polym. Sci., 2007, 32, 534-595.

6 L. Wu, L. Zong, H. Ni, X. Liu, W. Wen, L. Feng, J. Cao, X. Qi, Y. Ge and S. Shen, Biomater. Sci., 2019, 7, 2134-2143.

7 M. Yan, J. Ge, W. Dong, Z. Liu and P. Ouyang, Biochem. Eng. J., 2006, 30, 48-54.

8 W. Yuan and X. Chen, $R S C$ Adv. , 2016, 6, 6802-6810. 
9 A. Halperin, M. Kröger and F. M. Winnik, Angew. Chem., Int. Ed., 2015, 54, 15342-15367.

10 Q. Zhang, C. Weber, U. S. Schubert and R. Hoogenboom, Mater. Horiz., 2017, 4, 109-116.

11 M. Sponchioni, U. C. Palmiero and D. Moscatelli, Mater. Sci. Eng., C, 2019, 102, 589-605.

12 W. Fu, C. Luo, E. A. Morin, W. He, Z. Li and B. Zhao, ACS Macro Lett., 2017, 6, 127-133.

13 M. Li, X. He, Y. Ling and H. Tang, Polymer, 2017, 132, 264272.

14 Y. Kohno, S. Saita, Y. Men, J. Yuan and H. Ohno, Polym. Chem., 2015, 6, 2163-2178.

15 H. Tao, E. Galati and E. Kumacheva, Macromolecules, 2018, 51, 6021-6027.

16 J. Seuring and S. Agarwal, Macromol. Rapid Commun., 2012, 33, 1898-1920.

17 Z. Zhang, H. Li, S. Kasmi, S. Van Herck, K. Deswarte, B. N. Lambrecht, R. Hoogenboom, L. Nuhn and B. G. De Geest, Angew. Chem., Int. Ed., DOI: 10.1002/anie.201900224.

18 N. Shimada, M. Nakayama, A. Kano and A. Maruyama, Biomacromolecules, 2013, 14, 1452-1457.

19 N. Shimada, M. Saito, S. Shukuri, S. Kuroyanagi, T. Kuboki, S. Kidoaki, T. Nagai and A. Maruyama, ACS Appl. Mater. Interfaces, 2016, 8, 31524-31529.

20 J. Seuring and S. Agarwal, Macromolecules, 2012, 45, 39103918.

21 B. A. Pineda-Contreras, H. Schmalz and S. Agarwal, Polym. Chem., 2016, 7, 1979-1986.

22 A. Augé, D. Fortin, X. Tong and Y. Zhao, Polym. Chem., 2018, 9, 4660-4673.

23 A. Palanisamy and S. A. Sukhishvili, Macromolecules, 2018, 51, 3467-3476.

24 F. Käfer, A. Lerch and S. Agarwal, J. Polym. Sci., Part A: Polym. Chem., 2017, 55, 274-279.

25 Y. Deng, F. Käfer, T. Chen, Q. Jin, J. Ji and S. Agarwal, Small, 2018, 14, 1802420.

26 N. Shimada, H. Ino, K. Maie, M. Nakayama, A. Kano and A. Maruyama, Biomacromolecules, 2011, 12, 3418-3422.

27 N. Shimada, T. Sasaki, T. Kawano and A. Maruyama, Biomacromolecules, 2018, 19, 4133-4138.

28 T. Masuda, N. Shimada, T. Sasaki, A. Maruyama, A. M. Akimoto and R. Yoshida, Angew. Chem., Int. Ed., 2017, 56, 9459-9462.

29 M. Ohshio, K. Ishihara, A. Maruyama, N. Shimada and S. Yusa, Langmuir, DOI: 10.1021/acs.langmuir.9b00849.

30 J. Ning, K. Kubota, G. Li and K. Haraguchi, React. Funct. Polym., 2013, 73, 969-978.
31 N. Wang, B. T. Seymour, E. M. Lewoczko, E. W. Kent, M.-L. Chen, J.-H. Wang and B. Zhao, Polym. Chem., 2018, 9, 5257-5261.

32 A. Imajo, T. Ito, T. Nousou and K. Shiraishi, 2016, 50, 1-6.

33 N. Morimoto, K. Muramatsu, S. M. Nomura and M. Suzuki, Colloids Surf., B, 2015, 128, 94-99.

34 L. Chen, Y. Honma, T. Mizutani, D. J. Liaw, J. P. Gong and Y. Osada, Polymer, 2000, 41, 141-147.

35 H. Willcock, A. Lu, C. F. Hansell, E. Chapman, I. R. Collins and R. K. O'Reilly, Polym. Chem., 2014, 5, 1023-1030.

36 J. Niskanen and H. Tenhu, Polym. Chem., 2017, 8, 220-232.

37 F. Wang, J. Yang and J. Zhao, Polym. Int., 2015, 64, 9991005.

38 N. M. Nizardo, D. Schanzenbach, E. Schönemann and A. Laschewsky, Polymers, 2018, 10, 325-347.

39 C. J. Fennell, A. Bizjak, V. Vlachy and K. A. Dill, J. Phys. Chem. B, 2009, 113, 6782-6791.

40 R. Ferrari, Y. Yu, M. Morbidelli, R. A. Hutchinson and D. Moscatelli, Macromolecules, 2011, 44, 9205-9212.

41 R. Ferrari, L. Talamini, M. B. Violatto, P. Giangregorio, M. Sponchioni, M. Morbidelli, M. Salmona, P. Bigini and D. Moscatelli, Mol. Pharm., 2017, 14, 124-134.

42 U. Capasso Palmiero, L. Morosi, M. Lupi, M. Ponzo, R. Frapolli, M. Zucchetti, P. Ubezio, M. Morbidelli, M. D'Incalci, E. Bello and D. Moscatelli, Macromol. Biosci., 2018, 18, 1800164.

43 K. P. Ananthapadmanabhan, E. D. Goddard, N. J. Turro and P. L. Kuo, Langmuir, 1985, 1, 352-355.

44 M. Sponchioni, U. Capasso Palmiero, N. Manfredini and D. Moscatelli, React. Chem. Eng., 2019, 4, 436-446.

45 J. Ladd, Z. Zhang, S. Chen, J. C. Hower and S. Jiang, Biomacromolecules, 2008, 9, 1357-1361.

46 Y. Li, R. Liu, Y. Shi, Z. Zhang and X. Zhang, Theranostics, 2015, 5, 583-596.

47 K. P. García, K. Zarschler, L. Barbaro, J. A. Barreto, W. O'Malley, L. Spiccia, H. Stephan and B. Graham, Small, 2014, 10, 2516-2529.

48 U. Capasso Palmiero, M. Maraldi, N. Manfredini and D. Moscatelli, Biomacromolecules, 2018, 19, 1314-1323.

49 M. Sponchioni, R. Ferrari, L. Morosi and D. Moscatelli, J. Polym. Sci., Part A: Polym. Chem., 2016, 54, 2919-2931.

50 R. Ferrari, M. Sponchioni, M. Morbidelli and D. Moscatelli, Nanoscale, 2018, 10, 22701-22719.

51 N. J. Ganson, T. J. Povsic, B. A. Sullenger, J. H. Alexander, S. L. Zelenkofske, J. M. Sailstad, C. P. Rusconi and M. S. Hershfield, J. Allergy Clin. Immunol., 2016, 137, 1610-1613. 